\title{
Shark Fin Electrocardiogram: A Deadly Electrocardiogram Pattern in ST-Elevation Myocardial Infarction (STEMI)
}

\author{
Amit K. Jaiswal ${ }^{1}$, Sunil Shah ${ }^{2,3}$ \\ 1. Internal Medicine, Ministry of Health, Male', MDV 2. Medicine, Ministry of Health, Male', MDV 3. Internal medicine, \\ California Institute of Behavioral Neurosciences \& Psychology, California, USA
}

Corresponding author: Amit K. Jaiswal, jaiswalamitj@gmail.com

\begin{abstract}
Shark fin electrocardiographic (ECG) pattern, also known as 'Lambda-wave', 'giant R waves', or 'triangular QRS-ST-T waveform' is a dangerous ECG pattern associated with ST-elevation myocardial infarction (STEMI). It is formed by the fusion of QRS, ST, and T waves and predicts the high risk of mortality due to cardiogenic shock and ventricular fibrillation. The management should be aggressive with reperfusion via thrombolysis or percutaneous intervention, ideally in the intensive care unit with ventricular assist devices. This ECG pattern may be misdiagnosed as wide complex tachycardia or the ECG changes of hyperkalemia. Thus, differentiating it from other conditions causing similar ECG changes and prompt management is highly important to save the patient from serious complications. Here we have presented a case of STEMI with shark fin ECG associated with pulmonary edema (Killip class III acute myocardial infarction).
\end{abstract}

Review began 06/05/2021 Review ended 06/15/2021 Published 06/28/2021

\section{() Copyright 2021}

Jaiswal et al. This is an open access article distributed under the terms of the Creative Commons Attribution License CC-BY 4.0., which permits unrestricted use, distribution, and reproduction in any medium, provided the original author and source are credited.
Categories: Cardiology, Emergency Medicine, Internal Medicine

Keywords: shark fin, lambda wave, giant $r$ wave, triangular qrs-st-t waveform, stemi

\section{Introduction}

Shark Fin pattern is an uncommon but high-risk electrocardiographic (ECG) pattern formed by fusion of QRS, ST-segment, and T waves [1]. Myocardial infarction with shark fin ECG pattern most commonly involves occlusion of the left main coronary artery and is associated with a high risk of death due to cardiac arrest and cardiogenic shock [2]. Therefore, aggressive management is needed in such patients. It can be easily mistaken for other conditions such as a wide complex tachycardia and hyperkalemia-associated changes in ECG. Early recognition and differentiating it from other causes of similar changes in ECG is important to prevent higher rates of acute complications such as cardiogenic shock and ventricular arrhythmia.

\section{Case Presentation}

A 48-year-old male presented to the emergency department (ED) with complaints of chest pain, breathing difficulty, and vomiting for two to three hours. He also complained of sweating and orthopnea. On examination, his blood pressure was $130 / 70 \mathrm{mmHg}$, pulse rate 120 beats/minute, respiratory rate 24 cycles/min, temperature 37-degree centigrade, and oxygen saturation $86 \%$ in room air. There were coarse crepitations on both the lungs. He had a history of hypertension and hyperlipidemia under irregular medication due to poor adherence to treatment. He had a 20-pack-year smoking history, but he denied intake of alcohol and illicit drug use.

He was placed in a reclined position at a 45-degree angle. Oxygen was given at the rate of 6 liters/minute to maintain oxygen saturation of more than $96 \%$. The ECG done at the emergency department (ED) is shown in Figure 1.

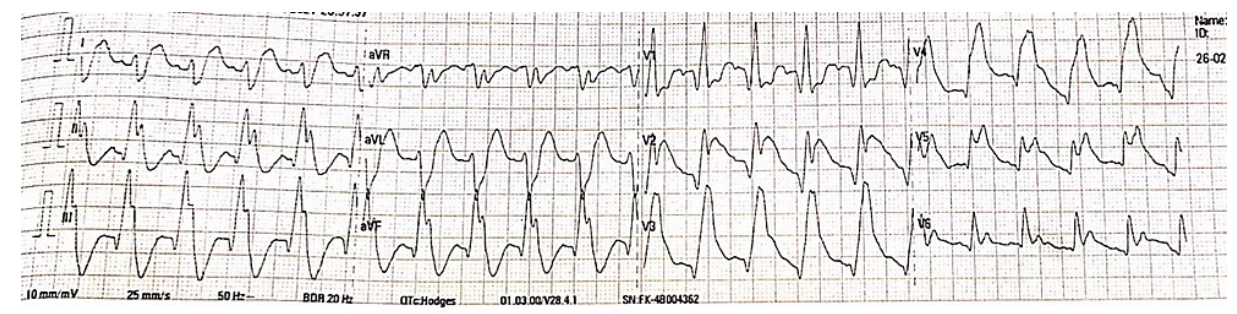

FIGURE 1: ECG at Presentation 


\section{Cureus}

A comprehensive metabolic panel along with serum potassium level was within normal limits. The diagnosis of extensive ST-elevation myocardial infarction (STEMI) was made. Aspirin $300 \mathrm{mg}$ and atorvastatin $80 \mathrm{mg}$ were given immediately. Due to the unavailability of recombinant tissue plasminogen activator (rTPA), streptokinase was given for thrombolysis. The ECG at 90 minutes post thrombolysis is as shown in Figure 2.

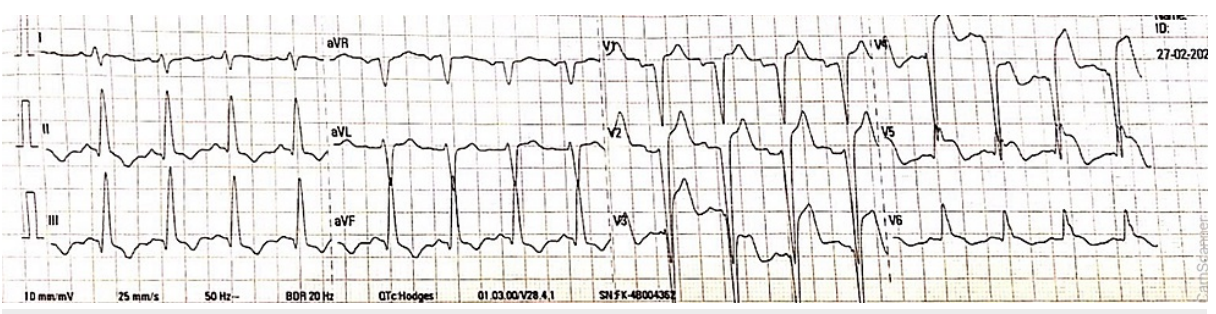

FIGURE 2: ECG After 90 Minutes of Thrombolysis

The patient had decreased oxygen saturation $(<90 \%)$ even after $2 \mathrm{mg}$ of morphine three times and $140 \mathrm{mg}$ of furosemide. Continuous positive airway pressure (CPAP) was then administered to maintain saturation. Once he was resuscitated, he was sent to the higher center for percutaneous coronary intervention (PCI). In the higher center, coronary angiography was done, and a critical occlusion of the left anterior descending coronary artery was relieved with thrombectomy and stent placement. His condition was stable post this.

\section{Discussion}

Shark Fin ECG (SFE) pattern is a high-risk giant wave (amplitude $\geqslant 1 \mathrm{mV}$ ) ECG pattern associated with a large burden of myocardial ischemia [3]. It is also known as 'Lambda-wave', 'giant R waves', or 'triangular QRS-STT waveform' [4]. This ECG pattern consists of blurring of the QRS complex due to its fusion with the STsegment and the T-wave showing a triangular lambda pattern where a positive deflection in the leads suggests ischemia in corresponding areas [1]. According to one study done by Cipriani et al. [3], only $1.4 \%$ of patients admitted due to STEMI developed this ECG pattern, illustrating its rarity.

SFE is usually associated with the occlusion/sub-occlusion of the left main coronary artery, left anterior descending artery, or proximal right coronary artery, especially in the absence of collateral coronary circulation [3]. The electrogenesis of this wave in patients with acute myocardial infarction (AMI) remains to be elucidated. The expansion of the left ventricular cavity during acute myocardial infarction (Brody's hypothesis), the increase in tissue resistance affecting myocardial conductivity factor ("the solid angle theorem”), or the difference in response of epicardial and endocardial action potentials are some of the hypotheses for the genesis of this waveform [5-7].

The SFE associated AMI is more frequently complicated by cardiogenic shock or cardiac arrest; however, our case was presented with pulmonary edema (Killip class III). Killip classification of acute myocardial infarction is as shown in Table 1 [8].

\section{Killip Class Characteristic Features}

Class I No clinical evidence of heart failure

Class II Findings consistent with mild to moderate heart failure (third heart sound S3, lung rales, or jugular venous distension)

Class III Pulmonary edema

Class IV Cardiogenic shock or arterial hypotension (SBP $<90 \mathrm{mmHg})$

\section{TABLE 1: Killip Classification of Acute Myocardial Infarction}

SBP $=$ Systolic Blood Pressure

This ECG finding can be confused with wide complex tachycardia, ECG changes in myopericarditis, and hyperkalemia. Furthermore, it may be confused with the tombstoning ECG pattern of STEMI seen in 10-15\% of the patients. The features of tombstoning ECG differ from those of Shark Fin pattern regarding the presence of an elevated, convex upward ST-segment that connects a small and short R wave with a higher and broader T wave [9].

The management of the AMI with SFE should be prompt and more aggressive in an intensive care unit 
equipped with ventricular assist devices to address the impending cardiogenic shock and increase the survival rate. With both electrical and hemodynamic instability, this ECG pattern is associated with poor prognosis in patients with STEMI. Most of them experience ventricular fibrillation and/or cardiogenic shock [3]. The increased frequency of development of ventricular fibrillation, especially in the acute phase of these patients, causes a high risk of mortality [3].

\section{Conclusions}

The shark fin waveform is an uncommon but high-risk ECG pattern of STEMI which should be diagnosed early and differentiated from other conditions causing similar waveforms such as wide-complex tachycardia and hyperkalemia. The shark fin STEMI is associated with a high risk of both ventricular fibrillation (VF) and cardiogenic shock, accounting for increased in-hospital mortality. Thus, it requires aggressive management, including mechanical circulatory support.

\section{Additional Information \\ Disclosures}

Human subjects: Consent was obtained or waived by all participants in this study. Conflicts of interest: In compliance with the ICMJE uniform disclosure form, all authors declare the following: Payment/services info: All authors have declared that no financial support was received from any organization for the submitted work. Financial relationships: All authors have declared that they have no financial relationships at present or within the previous three years with any organizations that might have an interest in the submitted work. Other relationships: All authors have declared that there are no other relationships or activities that could appear to have influenced the submitted work.

\section{References}

1. Hasibuan FS, Intan RE, Wilujeng HRT, Octora TN, Dharmajati BB, Gandi P, Alkaff FF: Triangular QRS-ST-T waveform electrocardiographic pattern in acute myopericarditis: a case report from a limited-resources hospital. Am J Case Rep. 2020, 21:e926360. 10.12659/AJCR.926360

2. Fiol M, Carrillo A, Rodríguez A, Pascual M, Bethencourt A, Bayés de Luna A: Electrocardiographic changes of ST-elevation myocardial infarction in patients with complete occlusion of the left main trunk without collateral circulation: differential diagnosis and clinical considerations. J Electrocardiol. 2012, 45:487-90. 10.1016/j.jelectrocard.2012.05.001

3. Cipriani A, D'Amico G, Brunello G, et al.: The electrocardiographic "triangular QRS-ST-T waveform" pattern in patients with ST-segment elevation myocardial infarction: incidence, pathophysiology and clinical implications. J Electrocardiol. 2018, 51:8-14. 10.1016/j.jelectrocard.2017.08.023

4. Miranda JM, de Oliveira WS, de Sá VP, de Sá IF, Neto NO: Transient triangular QRS-ST-T waveform with good outcome in a patient with left main coronary artery stenosis: A case report. J Electrocardiol. 2019, 54:87-9. 10.1016/j.jelectrocard.2019.02.002

5. Brody DA: A theoretical analysis of intracavitary blood mass influence on the heart-lead relationship . Circ Res. 1956, 4:731-8. 10.1161/01.res.4.6.731

6. Cordeiro JM, Mazza M, Goodrow R, Ulahannan N, Antzelevitch C, Di Diego JM: Functionally distinct sodium channels in ventricular epicardial and endocardial cells contribute to a greater sensitivity of the epicardium to electrical depression. Am J Physiol Heart Circ Physiol. 2008, 295:H154-62. 10.1152/ajpheart.01327.2007

7. Childers R: R wave amplitude in ischemia, injury, and infarction. Plenary address . J Electrocardiol. 1996, 29:171-178. 10.1016/s0022-0736(96)80058-6

8. Killip T 3rd, Kimball JT: Treatment of myocardial infarction in a coronary care unit. A two year experience with 250 patients. Am J Cardiol. 196720, 457-464. 10.1016/0002-9149(67)90023-9

9. Guo XH, Yap YG, Chen LJ, Huang J, Camm AJ: Correlation of coronary angiography with "tombstoning" electrocardiographic pattern in patients after acute myocardial infarction. Clin Cardiol. 2000, 23:347-52. $10.1002 /$ clc. 4960230508 